
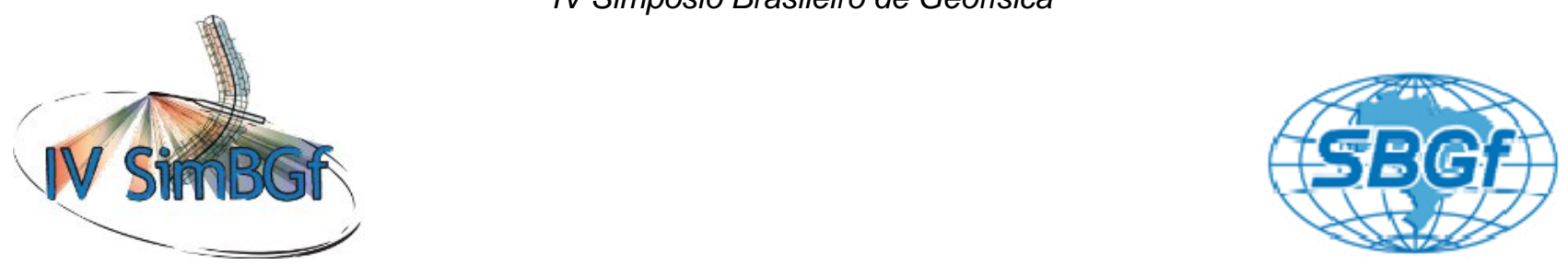

\title{
Titulo: Classificação de Fácies Através da Aplicação da Transformada Wavelet em Dados de Perfilagem Geofísica
}

Autores: Alexandre Cruz Sanchetta, Bruno César Zanardo Honório e Alexandre Campane Vidal. Instituição: Universidade Estadual de Campinas.

Copyright 2010, SBGf - Sociedade Brasileira de Geofísica

Este texto foi preparado para a apresentação no IV Simpósio Brasileiro de Geofísica, Brasília, 14 a 17 de novembro de 2010. Seu conteúdo foi revisado pelo Comitê Técnico do IV SimBGf, mas não necessariamente representa a opinião da SBGf ou de seus associados. É proibida a reprodução total ou parcial deste material para propósitos comerciais sem prévia autorização da SBGf.

\begin{abstract}
In this study we present an approach to facies classification based on well log responses and wavelet transform. The preliminary results presented in this study address the confiability of the method and indicate that statistical features extracted from wavelet analysis works well in the context of facies classification.
\end{abstract}

\section{Introdução}

Com o aumento da complexidade do processo de exploração de hidrocarbonetos, torna-se crítica a compreensão das propriedades que governam as características dos reservatórios. Dentro desse contexto, a base de dados inclui perfis geofísicos, testemunhos, dados sísmicos dentre outros. Campos maduros com anos de produção geralmente possuem um vasto número de poços dos quais, por meio de seus perfis, são extraídos diversos atributos. Devido à inviabilidade econômica, somente alguns poucos poços possuem testemunhos.

Dessa forma, técnicas que possibilitem a classificação de maneira robusta a partir de dados de perfis geofísicos podem melhorar o processo de caracterização de reservatórios e, com isso, a obtenção de um modelo geológico mais preciso. Na maioria das vezes, essa classificação se baseia nas informações e nas correlações entre os diferentes tipos de perfis. Serra, em 1984, introduziu o conceito de eletrofácies, o qual descreve a rocha em termos das características dos perfis (Serra, et al., 1984). Outras metodologias se baseiam em técnicas estatísticas multivaridas (Rosa, 2006) e redes neurais(Sandham, et al., 2003).

Uma questão inerente à análise do sinal obtido como resposta do meio geológico está relacionada com o método empregado para tal. A representação de um sinal no domínio da frequência geralmente revela características que são de difícil visualização no domínio do tempo (Chakraborty, et al., 1995). Diversas são as ferramentas aplicadas para este propósito, sendo de uso comum a transformada de Fourier. Para muitos sinais a transformada de Fourier é extremamente útil, porém, em dados geofísicos, muitos dos fenômenos registrados apresentam um caráter não-estacionário. Do ponto de vista da transformada de Fourier, esta é uma característica indesejável, uma vez que ao identificar as frequências constituintes do sinal na transformação do domínio do tempo para o domínio da frequência, perdese totalmente a informação sobre a localização temporal (ou espacial) do evento. $\mathrm{Na}$ sua versão "janelada" (Windowed Fourier Transform - WFT), há a correspondência entre tempo-frequencia, porém uma vez escolhida a função janela, a resolução tempo-frequência é fixada para todo o sinal(Chakraborty, et al., 1995).

Com grande impacto a partir da década de 1980, a transformada wavelet (Wavelet Transform - WT) se mostrou uma técnica bem adequada para avaliar processos não-estacionários ou transitórios. Dentro do contexto da geofísica, Prokoph avaliou dados de rochafonte, via análise wavelet, identificando regiões de descontinuidades, bem como a presença de ciclos sedimentares (Prokoph, et al., 2000). Métodos baseados em wavelets têm sido recentemente aplicados no problema de segmentação de perfis de poços (Robail, et al., 1998; Robail, et al., 2001; Hruška, et al., 2009).

O presente trabalho propõe um método baseado na transformada wavelet contínua (Continuous Wavelet Transform - CWT) para a classificação de fácies a partir de perfis de poços.

\section{Metodologia/ Problema Investigado}

A idéia fundamental inerente à análise wavelet é examinar os dados de acordo com a escala. Segundo Farge, a análise wavelet atua como um microscópio matemático(Farge, et al., 1993). Através desse "microscópio", podem-se acessar as diferentes componentes do sinal e assim, interpretá-las individualmente. Diferentemente da análise de Fourier, a qual utiliza senos e cossenos como funções base, as wavelets são finitas e bem localizadas em ambos os domínios, tempo (ou espaço) e frequência (Wickerhauser, 1994).

Do ponto de vista matemático, a transformada wavelet contínua é dada por:

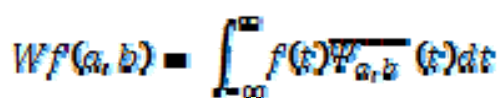

$(6 q, 1)$ 
Onde:

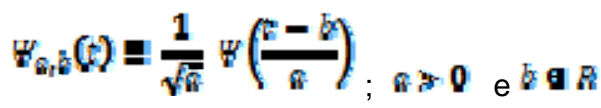

A função $W_{a, b}$ representa a família de wavelets, $\boldsymbol{a}$ o parâmetro de escala, $\boldsymbol{b}$ o parâmetro de localização da wavelet e $\overline{T_{G, b}}(t)$ denota o complexo conjugado de $W_{a, b}$. O parâmetro $\boldsymbol{a}$ dilata $(\boldsymbol{a}>1)$ ou contrai $(\boldsymbol{a}<1)$ a função $\mathbb{W}(t)$, enquanto que o parâmetro $b$ tem o efeito de analisar a função $f(t)$ em pontos distintos.

A aplicação da CWT resulta em diversos coeficientes $C^{\prime} \equiv \operatorname{VE} f(a, b)$, os quais medem a correlação entre o sinal e as wavelets utilizadas. Mais detalhadamente, na CWT a wavelet é comparada sucessivamente a diferentes seções do sinal, sendo que o produto de cada seção com a wavelet é uma nova função. A área delimitada por essa função são os coeficientes $\mathbf{C}$. Assim, quanto maior a similaridade entre a wavelet e a seção do sinal, maior será o coeficiente $C$ (Figura 1).

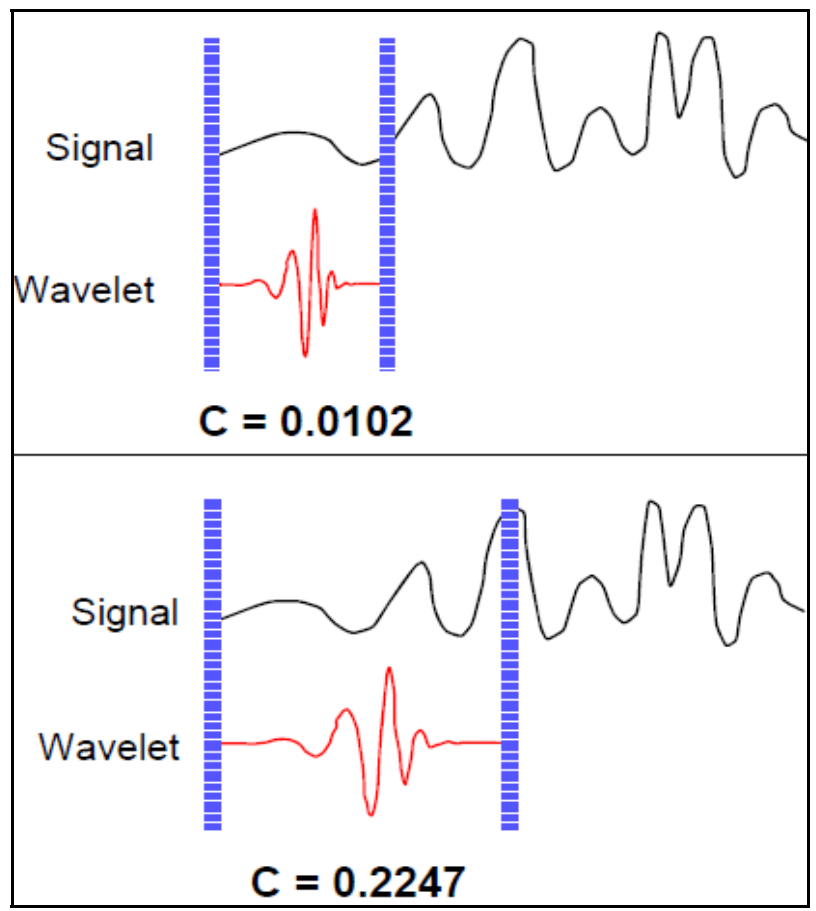

Figura 1: Ilustração da obtenção dos coeficientes via CWT(Misiti, et al., 1997)

Ao analisar toda a extensão do sinal para as diversas escalas de $\boldsymbol{a}$, tem-se um mapa de coeficientes, também conhecido como escalograma, que reflete as características do sinal (Figura 2).

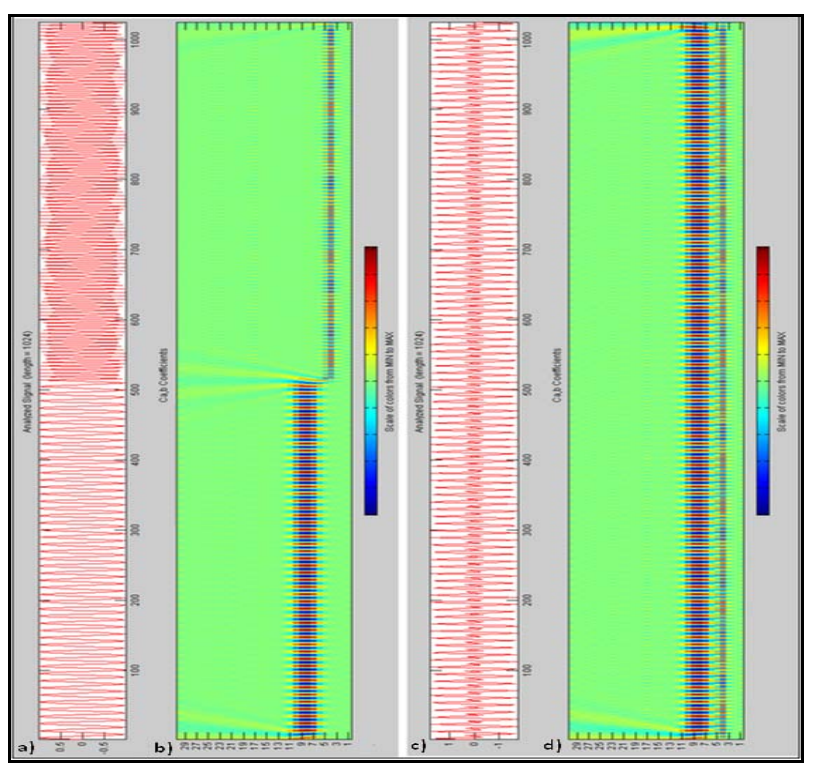

Figura 2: a) e c) Sinais analisados; b) e d) Mapa de coeficientes

Ao mapear onde e quais conteúdos de frequência ocorrem no escalograma, viabiliza-se a utilização de técnicas de reconhecimento de padrões que encontram essas codificações e, a partir de um conjunto de treino, reconheça e classifique novos conjuntos de dados.

No presente trabalho, utilizou-se o algoritmo de classificação supervisionada KNN (K-nearest neighbor, baseado na distância Euclidiana) que, basicamente, é treinado a partir do escalograma obtido dos perfis de poços e testemunhos, podendo assim classificar poços com ausência de testemunho. Para a validação do método, foram utilizados intervalos com testemunhos para a classificação e calculado a porcentagem de acerto.

\section{Resultados}

Para avaliar quantitativamente as respostas dadas pelo método, foram feitos gráficos que apresentam, no eixo das abcissas $(X)$, diferentes escolhas para o número de vizinhos do método K-NN e, no eixo das ordenadas ( $Y$ ), as respectivas porcentagens de acerto. Os gráficos são discretos e os valores têm seu real significado nos valores inteiros do eixo $X$. A base de dados se refere ao Campo de Namorado, Bacia de Campos. No presente estudo, utilizaram-se perfis Sônicos (DT) e, para validação do método, trechos dos poços que possuem testemunhos. Os pontos identificados com quadrados azuis fazem parte da classificação através de uma busca por padrões K-NN no conjunto de dados sem nenhum tipo de processamento. Os pontos identificados com quadrados verdes tiveram um processamento prévio através da transformada wavelet (wavelet Morlet), para então serem utilizados na classificação K-NN. O título 
das figuras é referente ao poço utilizado como o teste de classificação, sendo que para cada um deles, foram utilizados poços distintos como treino. A classificação é referente às características da rocha ser Reservatório, Não-Reservatório ou Possível Reservatório.

Para essa primeira análise, buscou-se classificar os dados presentes no poço NA01, utilizando o poço NA07 como treino (Figura 3).

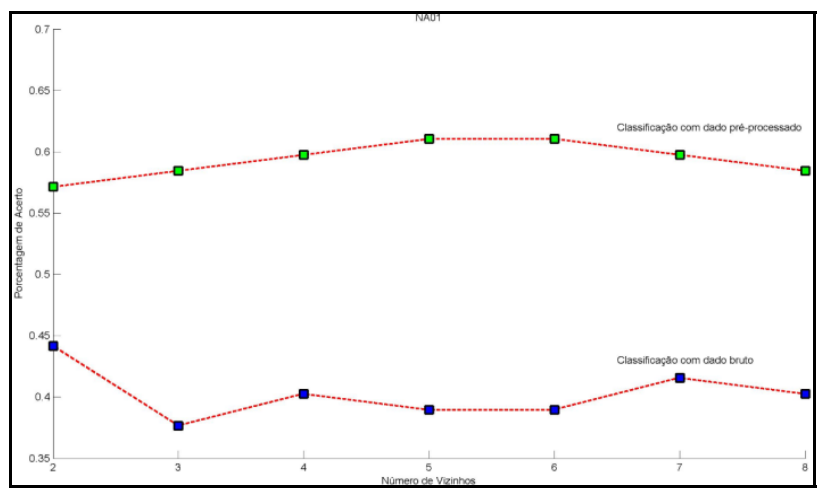

Figura 3: Classificação de Fácies do poço NA01

O resultado é expressivo, mostrando uma porcentagem de acerto por volta de $60 \%$ para os dados préprocessados, enquanto para os dados brutos, uma porcentagem de $40 \%$. O comportamento regular para o dado pré-processado indica também uma boa estrutura espacial dos dados, pois a taxa de acerto tende a permanecer na mesma região. Vale destacar que o poço NA07, que foi utilizado como treino, não apresenta todo o tipo de fácies presente no poço NA01, e mesmo assim, o teste foi satisfatório e com um resultado bem mais eficiente quando comparado com a classificação do dado bruto.

A Figura 4 apresenta o resultado da classificação do poço NA07, cujo treino se baseou nos dados do poço RJS42.

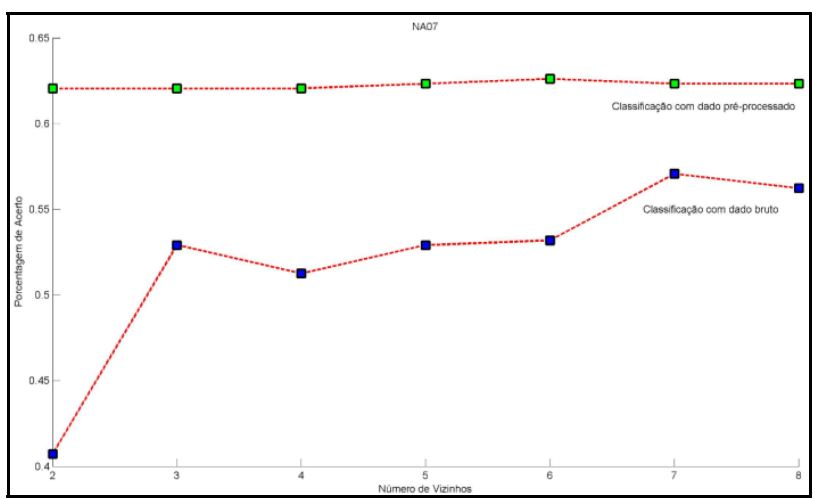

Figura 4: Classificação de Fácies do poço NA07

As diferenças entre as porcentagens de acerto do método são menores do que no primeiro teste, entretanto, a taxa de acerto com o pré-processamento ainda se mostra superior e mais regular do que a resposta para a classificação do dado bruto. Mais uma vez, a taxa de acerto está em cerca de $60 \%$.
Finalmente, para esse último teste, visou-se a classificação do poço RJS234, sendo o poço NA07 utilizado para o treino, assim como no primeiro teste. A Figura 5 mostra o resultado obtido.

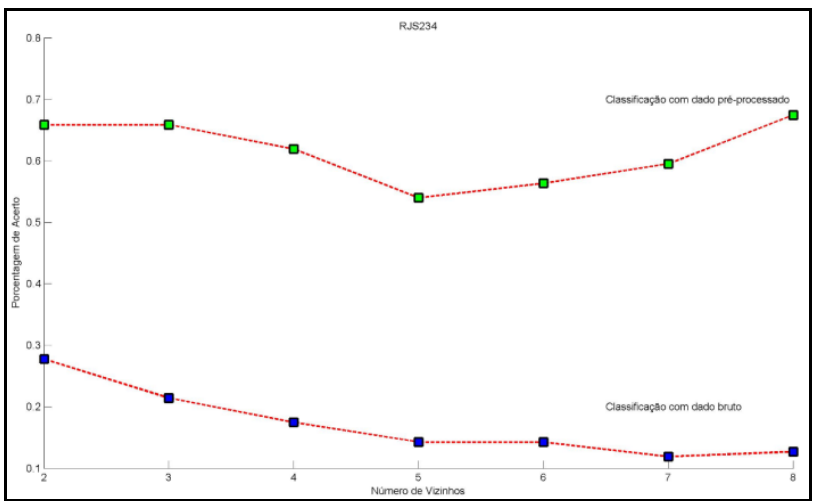

Figura 5: Classificação de Fácies do poço NA07

O resultado é mais promissor ainda que os testes anteriores, pois além da diferença pontual entre as taxas de acerto, o método com o pré-processamento através da análise wavelet atinge valores próximos a $70 \%$ de acerto. O comportamento um pouco irregular sugere uma complexidade espacial diferenciada dos outros testes.

A figura 6 ilustra o trecho com testemunho do poço NA01, bem como o resultado da classificação através do dado pré-processado e através do dado bruto:

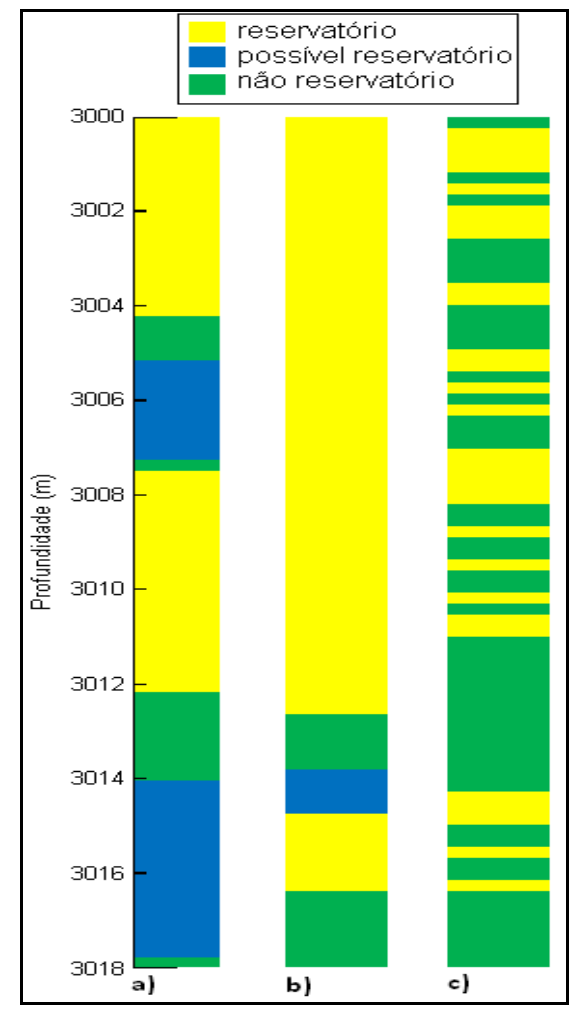

Figura 6: Fácies do poço NA01: a) Testemunho; b) Dado pré-processado; c) Dado bruto 


\section{Discussão e Conclusões}

Neste estudo apresentou-se um método de classificação de fácies baseada em dados de perfis geofísicos e na transformada wavelet. Os resultados preliminares mostram que o método empregado apresenta uma melhora significativa na previsão e classificação das litofácies quando é utilizado o pré-processamento via análise wavelet. A melhor classificação se deve à extensão na dimensionalidade do conjunto de dados. É possível conseguir maiores taxas de acerto quando se tratam de treinos dentro do mesmo poço, entretanto, para uma validação mais robusta do método, a opção do treino com um poço distinto do poço escolhido para a classificação se fez a mais adequada.

Além da taxa de acerto superior nos três testes, a regularidade apresentada nos resultados com préprocessamento sugere que a estrutura espacial dos dados tenha maior coerência para a classificação que o dado bruto, pois os dados sem pré-processamento têm maiores variações nos índices de acerto e apresentam um comportamento com padrão aleatório.

Uma taxa média de acerto superior a $60 \%$ somada com a regularidade das respostas encontradas evidenciam a potencialidade do método, representando um expressivo avanço quanto à classificação de características das rochas, considerando a pequena quantidade de dados utilizados como treino. Este estudo está em andamento, tendo em vista que existem diversos parâmetros passíveis de serem alterados, tais como a escolha da wavelet, o método de classificação ou as próprias variações do método K-NN, além da utilização de maior número de atributos.

\section{Agradecimentos}

Os autores agradecem à ANP - Agência Nacional de Petróleo e à Petrobras - Petróleo Brasileiro SA - pela disponibilização dos dados do Campo de Namorado utilizados no presente trabalho; aos pesquisadores Rodrigo Duarte Drummond e Emilson Pereira Leite pela revisão do texto e Evaldo Cesário Mundim pelas sugestões ao longo do projeto.

\section{Referências}

Chakraborty A. and Okaya D. - 1995 - Frequency-time decomposition of seismic data using wavelet-based methods - Geophysics, Vol. 60, No. 6, pp.1906-1916.

Farge M., Hunt Julian C. R. and Vassilicos J. C. - 1993 Wavelets, Fractals and Fourier Transforms: New Developments and New Applications - Oxford University Press.
Hruška, M.; Corea, W.; Seeburger, D.; Schweller, W. Crane, W.H. - 2009 - Automated Segmentation of Resistivity Image Logs Using Wavelet Transform - Math Geosci - 41: 703-716.

Misiti, M.; Misiti, Y.; Oppenheim, G.; Poggi, J.M. - 1997 Wavelet Toolbox ${ }^{\mathrm{TM}} 4$ - User's Guide.

Prokoph A. and Agterberg F.P. - 2000 - Wavelet Analysis of Well-Logging Data From Oil Source Rock, Egret Member, Offshore Eastern Canada - Bulletin of American Association of Petroleum Geologists, Vol. 84, No.10, pp. 1617-1632.

Robail F. and P. Rabiller - 1998 - Détection des ruptures dans les signaux diagraphiques - French Patent.

Robail F., Rabiller P. and Schulbaum L. - 2001 Sedimentary bodies identification using the phase coefficients of the wavelet transform - Society of Petrophysicists and Well Log Analysts - 42nd Annual Logging.

Rosa H. - 2006 - Estudo de Caracterização de Eletrofácies por meio de Perfis Geofísicos de Poços e de Amostras de Testemunhos Utilizando Estatística Multivariada - Tese de Doutorado em Ciências e Engenharia de Petróleo - Unicamp.

Sandham W. and Leggett M. - 2003 - Geophysical Application of Artificial Neural Networks and Fuzzy Logic Modern Approaches in Geophysics - Kluwer Academic Publishers.

Serra O. and Abbot H.T. - 1984 - The Contribution of Logging Data to Sedimentology and Stratigraphy, Society of Petroleum Engineers, p. 117-135. - 1984.

Wickerhauser M. V. - 1994 - Adapted Wavelet Analysis from Theory to Software (Wellesley, MA: A K Peters). 